\title{
Plano Oficial de Urbanización de Algarrobo, 1950: urbanismo saludable y visión de futuro para un balneario de la antigua Provincia de Santiago de Chile.
}

Official Plan of Urbanisation of Algarrobo, 1950: healthy urban planning and vision for a resort in the former province of Santiago de Chile.

María Isabel Pavez Reyes

\section{Filiación}

Académica del D. de Urbanismo de la F.A.U. de la Universidad de Chile.

mpavez@uchilefau.cl

\section{Resumen}

Se analiza el proyecto urbano aprobado en 1950 para la comuna de Algarrobo, Chile, a partir de su plano. A cargo de esta planificación estuvo la Sección de Urbanismo del Departamento de Arquitectura de la D.G.O.P., dirigida por el Arqto. Luis Muñoz M.

\section{Palabras clave}

Algarrobo, Chile, litoral central, planificación territorial, Luis Muñoz Maluschka, corredores verdes.

\section{Abstract}

This article analyzes the project for Algarrobo, Chile, approved in 1950 when Luis Muñoz M. was in charge of the plan management.

\section{Key words}

Algarrobo, Chile, central coast line, territorial planning, Luis Muñoz Maluschka, green corridors.

\section{Sumario}

1.-Desde Caleta a Puerto Menor.

2.- Proposiciones en "Algarrobo. Plano Oficial de Urbanización. Anteproyecto".

3.-Comentarios

Anexo

Fuentes de Información

\section{1.-Desde Caleta a Puerto Menor.}

Durante el siglo XIX, desde 1854, la "caleta de Algarrobo" pasó a ser "Puerto Menor" para efectos de transportar el trigo a Perú y a California. 
Ya en el siglo XX, en las décadas de 1930-1940 Carlos Alessandri Altamirano adquirió por compra la antigua hacienda Peñablanca de Algarrobo, pensando con espíritu visionario que éste sería el balneario del futuro en el litoral central, después de Viña del Mar. Fue así como empezó a urbanizar paulatinamente partes de su hacienda, comenzando por la nueva población de Algarrobo y otros como lo fue Santa Teresita y sus ampliaciones.

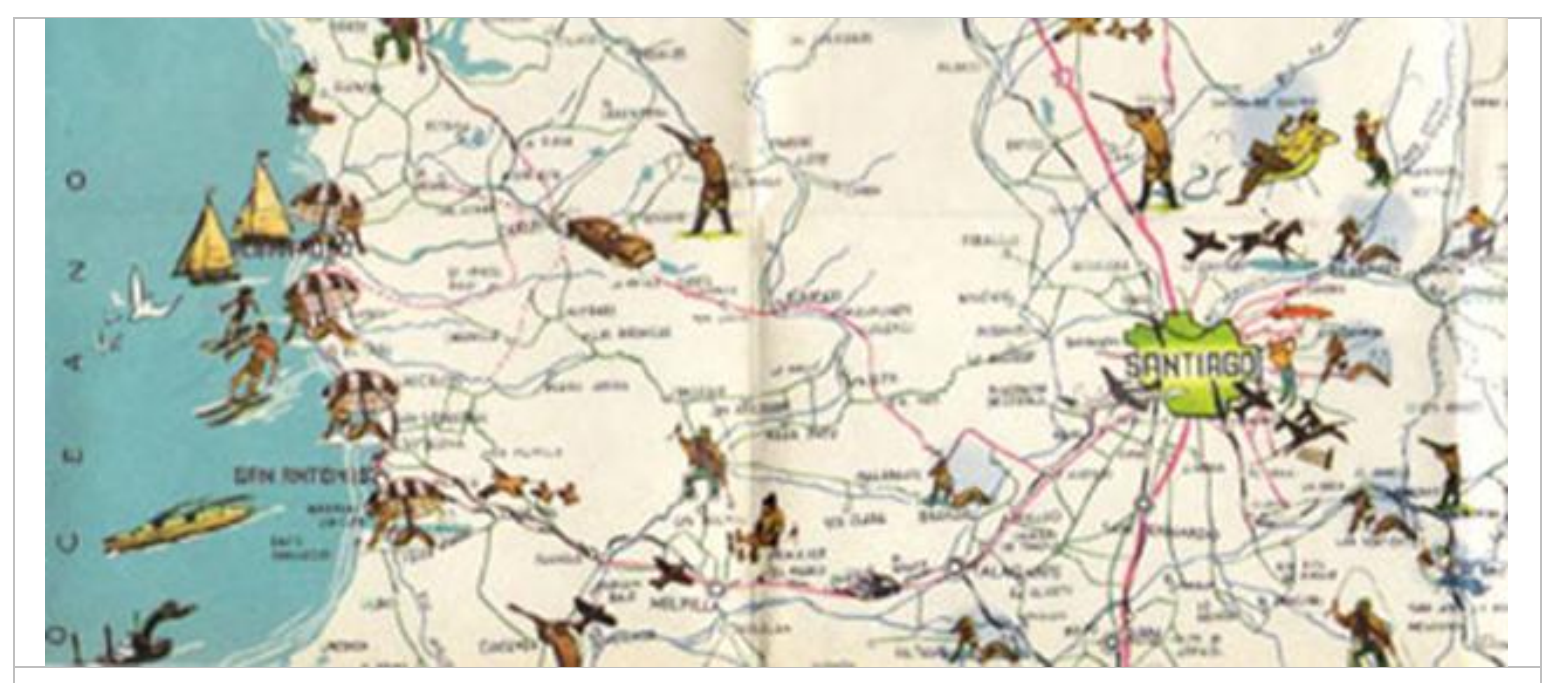

Fig.1. Costa frente a Santiago, entre Algarrobo y Rocas de Santo Domingo. Sección de mapa con la zona de Santiago y su frente costero, del mapa "Algunas referencias turísticas de las Provincias de Santiago, Valparaíso y Aconcagua". (Realización de Carlos Müller). En: EMPRESA DE FERROCARRILES DE ESTADO, Sección de Propaganda y Turismo: Guía del veraneante. Guía Anual de Turismo de la República de Chile. (Información de Oreste Plath y realización de Ketty Bravo). Santiago, 1957, 360 págs.

En 1945 (21 de noviembre, Ley N8.388), Algarrobo se constituyó en Comuna, y ante el desarrollo previsto para este lugar como balneario, comenzaron los estudios en la Sección de Urbanismo del Departamento de Arquitectura de la D.G.O.P. -dirigida por el Arqto. Luis Muñoz M.- para efectos de generar un "Plan Oficial de Urbanización" para Algarrobo. Esta ciudad pertenecía a la "Provincia de Santiago", antes de la regionalización de 1974.

A este proyecto, aprobado en todas las instancias durante 1950 (Alcalde de Carlos Alessandri Altamirano, -ver Fig.7-), corresponde el documento gráfico que presentamos y que, aparentemente, estuvo vigente hasta mediados de $1969^{1}$.

1 No se cuenta con la memoria del proyecto. 


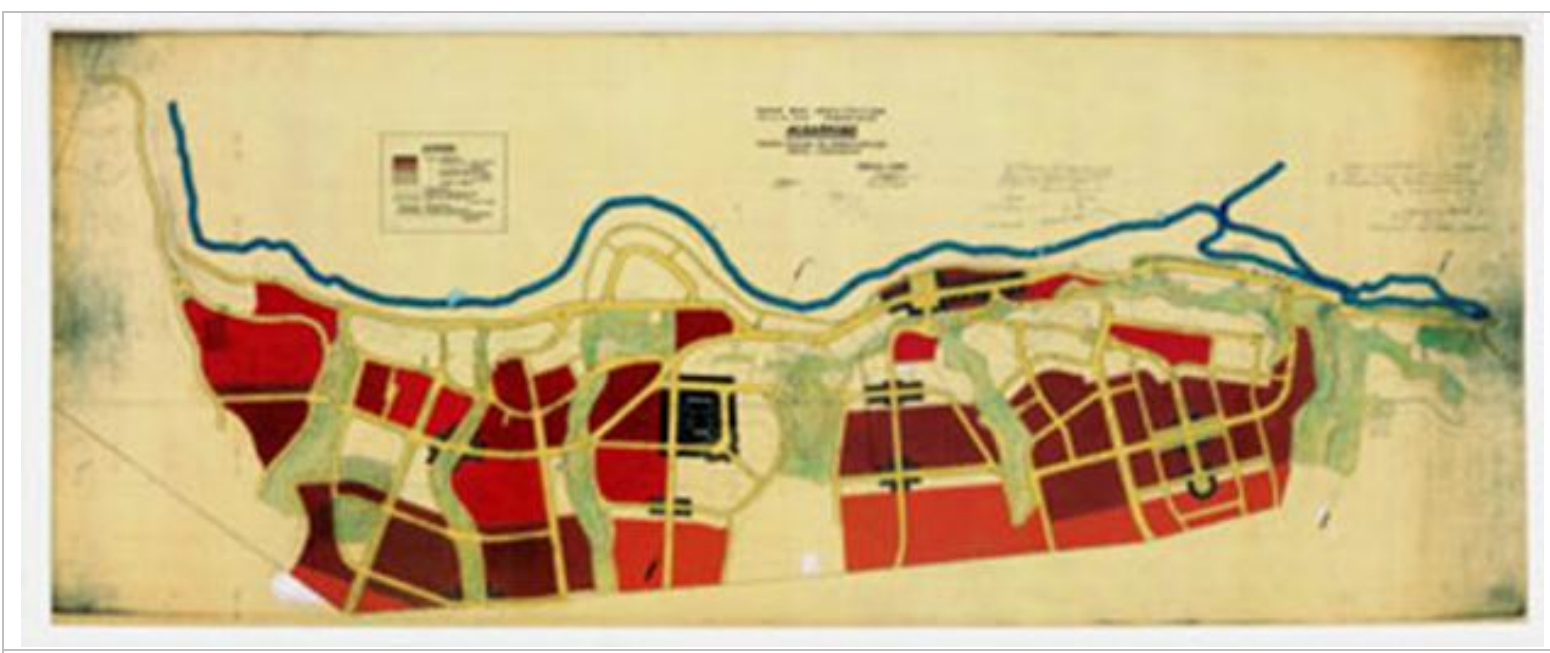

Fig.2. "Algarrobo. Plano Oficial de Urbanización. Anteproyecto". D.G.O.P., Departamento de Arquitectura, Sección Urbanismo, escala 1:2.000. (Firmado: Proyecto de C. Radbis (sic); $\mathrm{V}^{\circ} \mathrm{B}^{\circ}$ de Luis Muñoz Maluschka; firma del Alcalde Carlos Alessandri y Secretario Municipal (mayo de 1950). Aprobado por Decreto 1102 de 7 de julio de 1950, Firma del Sub-Secretario de Obras Públicas y Vías de Comunicación, timbre, (10 de septiembre de 1950), $(174 \mathrm{~cm} \times 70 \mathrm{~cm})$.

[Detalle de simbología y firmas de aprobaciones en ANEXO: Figs.7, 8, 9 y 10 en este escrito]. Fte.: Archivo del Departamento de Urbanismo de la F.A.U. de la Universidad de Chile, 2006.

\section{2.- Proposiciones en "Algarrobo. Plano Oficial de Urbanización. Anteproyecto".}

Básicamente observamos que en él se dispone:

- El establecimiento de un sistema de vías coordinado, donde se observa la instauración de una jerarquía de calles, con anchos variables según funcionalidad y restricciones del terreno;

- el reperfilamiento de caminos y huellas existentes, los que se integran al sistema;

- la apertura y continuación de nuevas vías teniendo en consideración las cotas de nivel y colaborando a definir bandas verdes paisajísticas en el estero "San Jerónimo", y las quebradas del "Lance Bravo", de "Los Claveles", de "Las Casas", de "Los Pescadores" y de "Las Tinajas";

- un parche verde lineal de $1.300 \mathrm{~m}$ de largo y 30-60 m de ancho, casi continuo, con la finalidad de unir por la parte baja, las áreas verdes del estero de San Jerónimo, y las quebradas de Lance Bravo, y de Los Claveles;

- un espacio verde de $220 \mathrm{~m} \times 105 \mathrm{~m}$, como remate de quebrada de Las Casas, junto a la costanera "Avda. del Mar", y como telón de fondo paisajístico interior de las playas del "Yachting" y del "Pejerrey"; 
- una vía costanera ( $22 \mathrm{~m}$ ), secundada por vías más o menos paralelas a ella en diversas alturas, de las cuales una de ellas intenta una centralidad dando lugar a diversos puentes, habida cuenta la necesidad de cruzar las diversas quebradas de Algarrobo;

- las vías colectoras principales que acometen perpendicularmente (aprox.) la costanera, se encuentran ubicadas por referencia a un módulo de 300-500 m aprox.;

- se observa especial consideración de las líneas de subdivisión predial para efectos del trazado de nuevas calles, localizando dichas líneas como ejes de las vías, o bien considerándolas coincidentes con una línea oficial de la vías;

- se establece zonas de equipamientos especiales, principales y de barrios, con un mercado localizado en el centro de Algarrobo, y zonas de uso mixto, residencial exclusivo, y también de vivienda económica en las zonas más altas;

- se considera una zona de futuro crecimiento hacia el sur-poniente. La zona nor-oriente tiene como limitación el estero San Jerónimo y el cementerio existente;

- en cuanto al límite urbano en la cota superior, se observa que unas 13 calles acometen perpendicularmente la línea imaginaria definida como límite, quedando como directrices para el futuro crecimiento y su acceso al borde costero.

\section{3.-Comentarios}

Cabe destacar que, en la actualidad, Algarrobo presenta diversos problemas de ordenamiento, entre ellos:

- uno que se refiere al tratamiento de las quebradas -que, desde el punto de vista técnico, siempre han sido consideradas como ductos de recepción de aguas lluvias y, desde el punto de vista la ecología del paisaje, como teselas o parches verdes relevantes, en nivel de refugio (hábitats de mayor calidad ambiental en el área urbana)-; se observa la llegada de personas que soslayando esto han construido sobre las quebradas interfiriendo, además, con las bajadas peatonales tradicionales por ellas;

- la construcción en altura, provocando interferencias a las vistas al mar, e incluso ocupando terrenos no adecuados para construir en altura;

- el desplazamiento del centro, lo que está muy poco claro, y por último,

- se ha invadido terrenos en áreas que históricamente se consideraron no ocupables, como son las dunas -una maravilla de la naturaleza-, y con ello se ha provocado rupturas en el balance hídrico de los terrenos interiores, además de la ruptura definitiva con el macro paisaje, especialmente en Algarrobo Norte.

Se observará que, en lo referido a las quebradas de Algarrobo, estos parches verdes de origen natural, considerados en el proyecto referido, tienen bordes curvilíneos, complejos y suaves, por lo cual favorecen el cruce de las especies que allí habitan, y por tanto, su desarrollo. Los límites que establecen los humanos tienden a ser rectos, simples y duros, lo cual favorece el movimiento de las especies cuando se trata de parches verdes. 
El mantenimiento de los corredores naturales que existan al interior de la ciudad y su interconexión con los refugios y escalones (parches menores) de creación humana es el aspecto más relevante de la aplicación de la ecología del paisaje a la ciudad. Vemos que el proyecto de Algarrobo para su futuro, aprobado hace 56 años, presentaba ya una clara conciencia y consideración al respecto.

En una ciudad balneario como es Algarrobo, donde fundamentalmente parte de la población de la metrópoli de Santiago busca recreación y mejor calidad de vida, el desarrollo articulado de la matriz espacial mediante una adecuada planificación urbanaregional, dará lugar a servicios ecológicos no sólo para el mantenimiento de la biodiversidad, sino también para la producción de aire y agua limpios, reciclaje y depuración del aire y aguas sucios, abatimiento de ruidos y aislamiento de fuentes contaminantes, morigeración de las islas térmica, de humedad y ventilación, entre otros.

Creemos que el territorio debe entenderse esencialmente como un bien común, pues contiene significados y relaciones con las comunidades que no pueden ser reducidos a mercaderías transables, tales como los servicios ambientales, los valores culturales, sentimientos de pertenencia e identidad cultural.

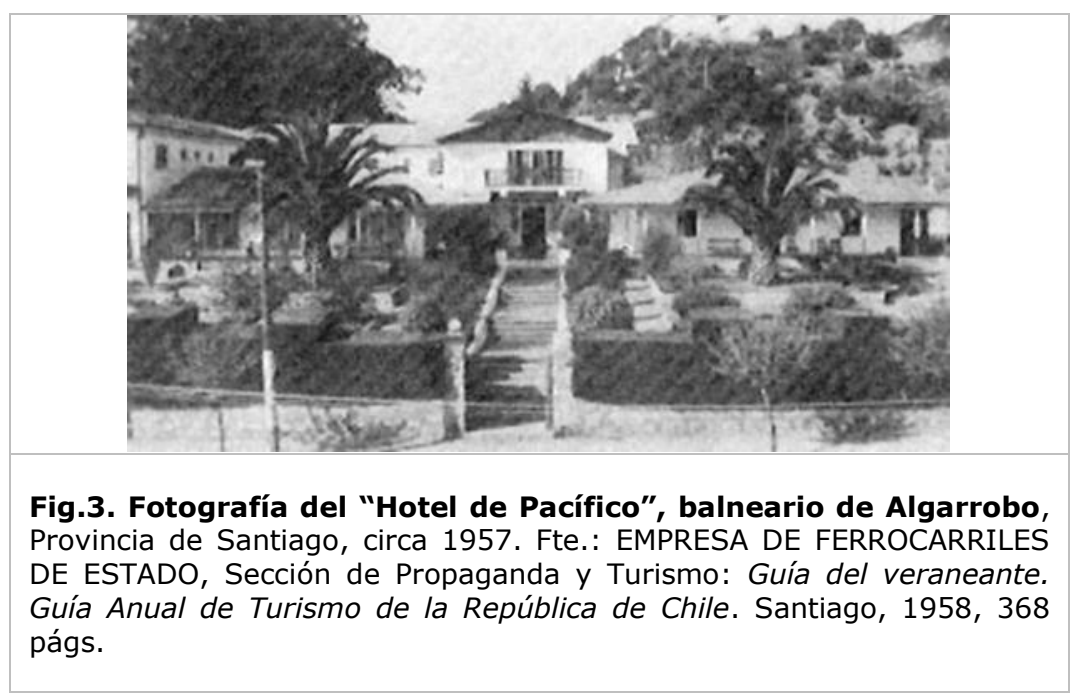




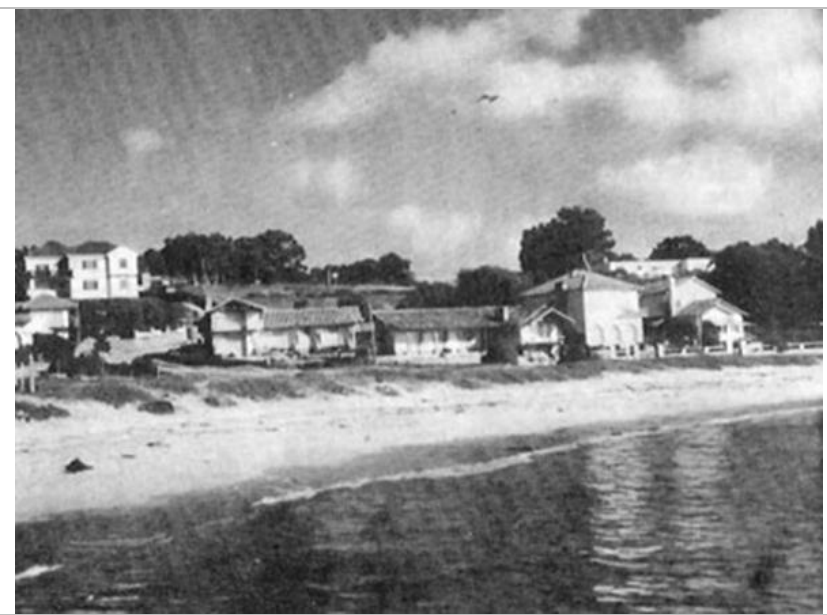

Fig.4. Vista de Algarrobo, balneario de la Provincia de Santiago, circa 1957. Fte.: EMPRESA DE FERROCARRILES DE ESTADO, Sección de Propaganda y Turismo: Guía del veraneante. Guía Anual de Turismo de la República de Chile. Santiago, 1958, 368 págs.

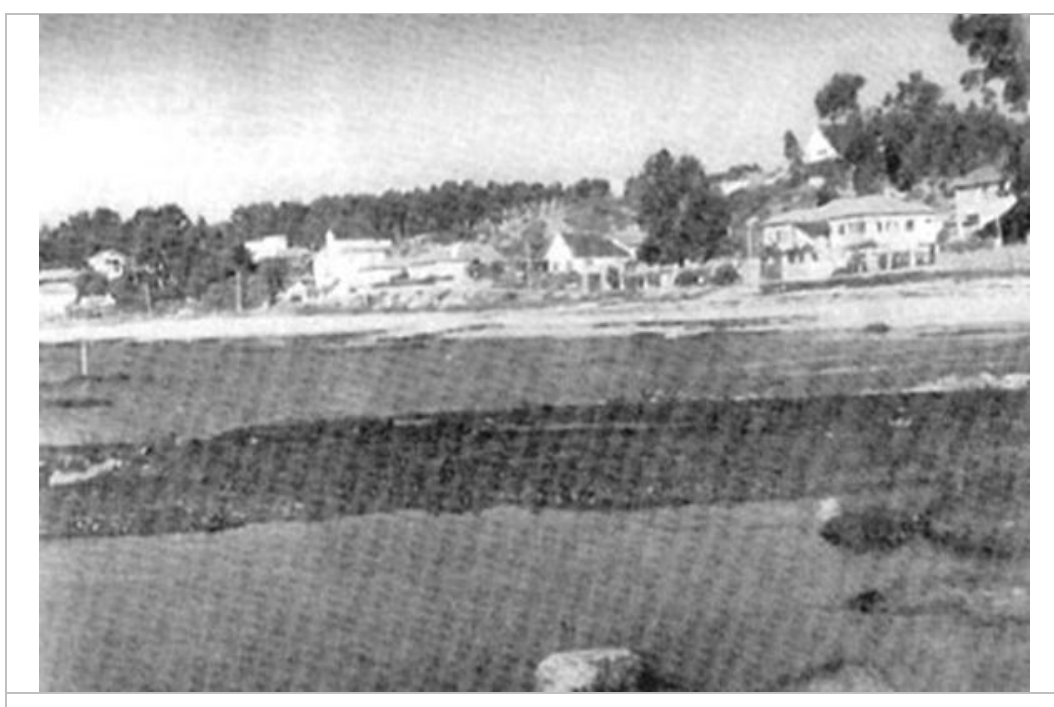

Fig.5. Vista de Algarrobo, balneario de la Provincia de Santiago, circa 1956. Fte.: EMPRESA DE FERROCARRILES DE ESTADO, Sección de Propaganda y Turismo: Guía del veraneante. Guía Anual de Turismo de la República de Chile. Santiago, 1957, 360 págs. 


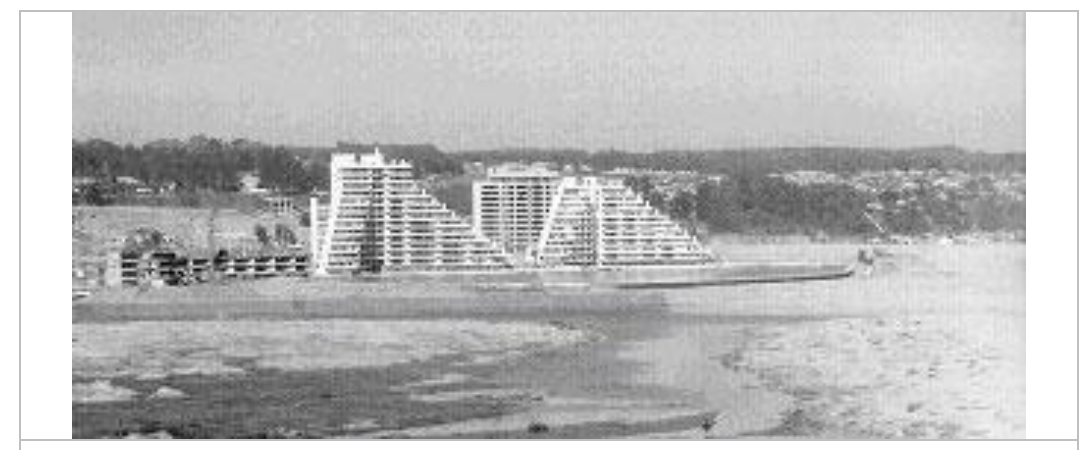

Fig.6. Megaproyecto turístico de segunda vivienda "San Alfonso del Mar", Algarrobo (al fondo Algarrobo tradicional). Sección de conjunto vacacional en construcción; el proyecto incluye una laguna artificial deportiva junto al océano Pacífico. Superficie del terreno: 25 Hás. en zona de playa y 56 Hás. en zona de cerro. Fte.: REVISTA CA CIUDAD/ARQUITECTURA, Órgano Oficial del Colegio de Arquitectos de Chile A.G. Enclaves vacacionales. No91, Santiago, oct., nov. dic., 1997. 


\section{Anexo}

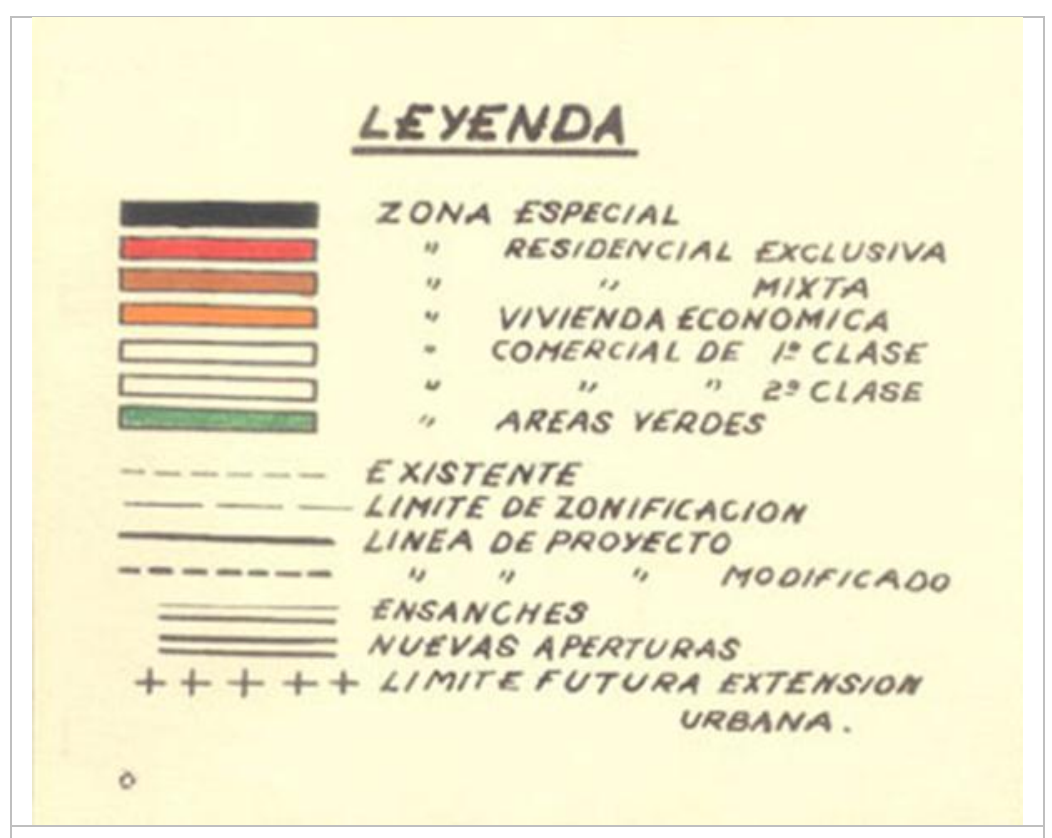

Fig.7. Detalle de cuadro de simbología del Plano Oficial de Urbanización de Algarrobo 1950.

D.G.O.P. DEP. ARQUITECTURA

SE C I I N URBANISMO

ALGARROBO

PLANO OFICIAL DE URBANIZACION ANTE - PROYECTO

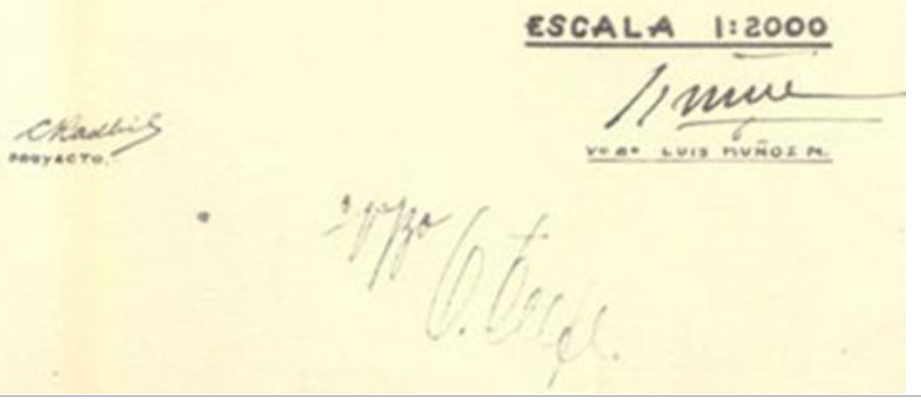

Fig.8. Detalle del $V^{\circ} B^{\circ}$ de Luis Muñoz Maluschka del Plano 
Oficial de Urbanización de Algarrobo 1950.

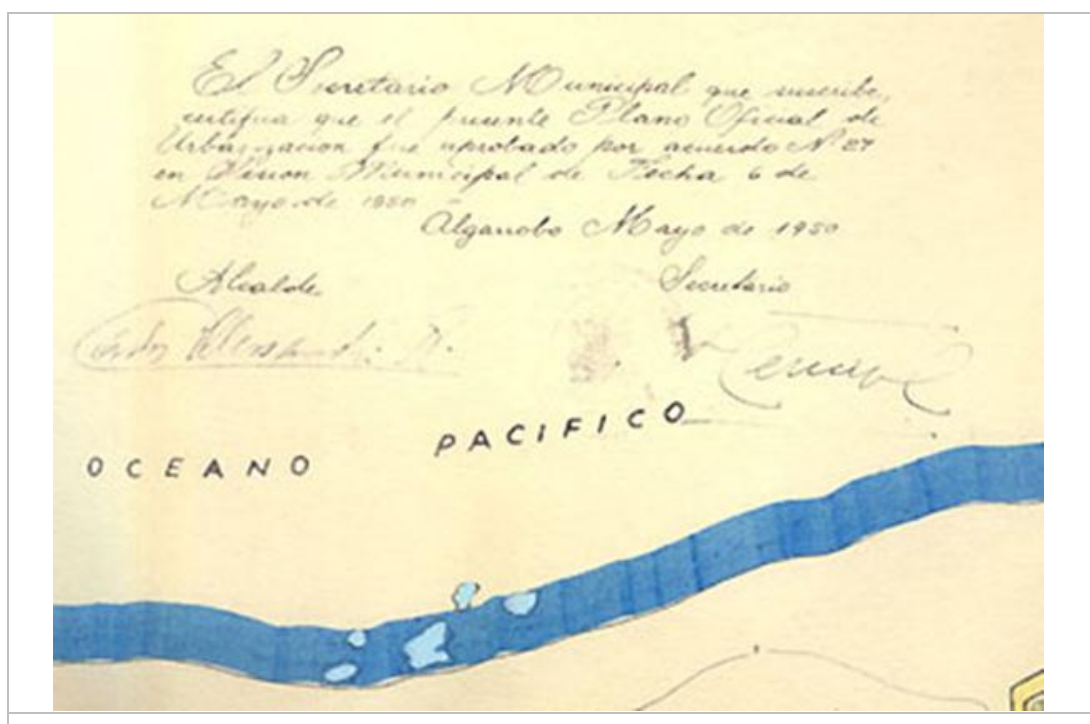

Fig.9. Detalle de firma del Alcalde Carlos Alessandri, del Plano Oficial de Urbanización de Algarrobo 1950.

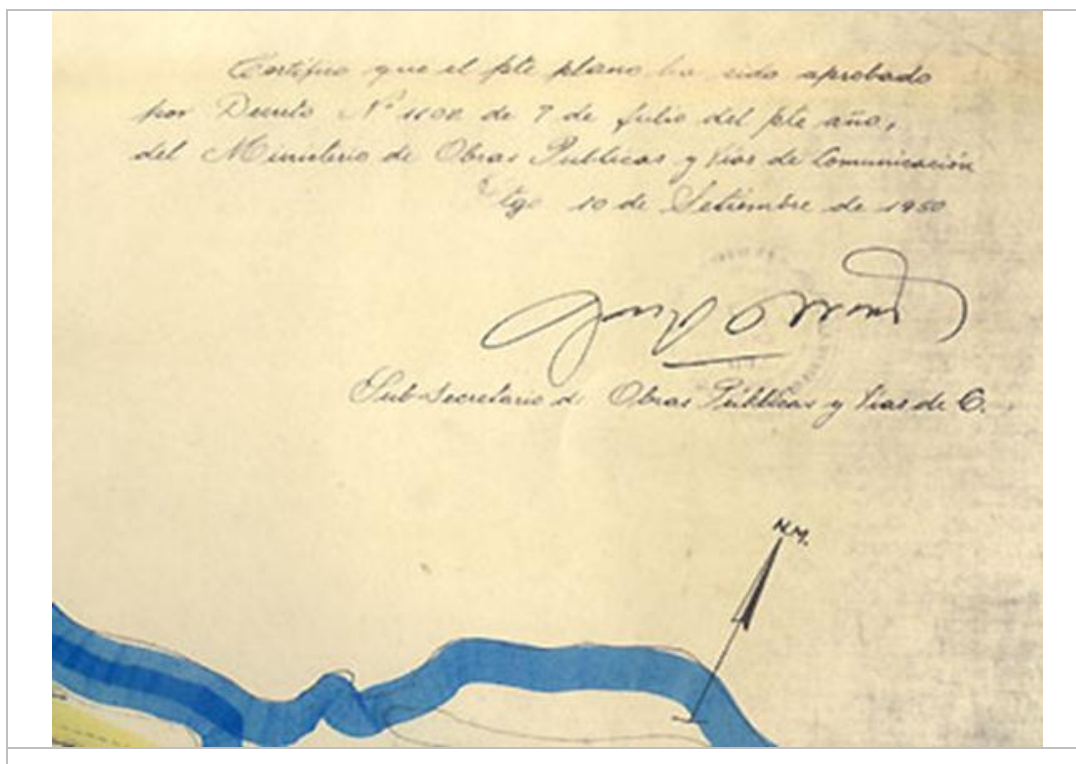

Fig. 10. Detalle de firma del Subsecretario de Obras públicas y Vías de Comunicación, del Plano Oficial de Urbanización de Algarrobo 1950.

\section{Fuentes de información}


UNIVERSIDAD DE CHILE, Departamento de Urbanismo de la Facultad de Arquitectura y Urbanismo: "Plano Oficial de Urbanización de Algarrobo 1950". [Copia matriz Escala original 1:2.000, en cartulina gruesa, coloreada a la témpera, en Archivo.

I. MUNICIPALIDAD DE ALGARROBO, página WEB, marzo 2006.

EMPRESA DE FERROCARRILES DE ESTADO, Sección de Propaganda y Turismo: Guía del veraneante. Guía Anual de Turismo de la República de Chile. Santiago, 1957, 360 págs.

EMPRESA DE FERROCARRILES DE ESTADO, Sección de Propaganda y Turismo: Guía del veraneante. Guía Anual de Turismo de la República de Chile. Santiago, 1958, 368 págs.

REVISTA CA CIUDAD/ARQUITECTURA, Órgano Oficial del Colegio de Arquitectos de Chile A.G. Enclaves vacacionales. No91, Santiago, oct., nov. dic., 1997.

ROMERO, Hugo, y Alexis VÁSQUEZ, 2004. "Pertenencia y significado del ordenamiento territorial en Chile". En: Seminario "El suelo en Chile: ¿entre el desarrollo y el mercado?, Comisión de Recursos Naturales, Bienes Nacionales y Medio Ambiente de la Cámara de Diputados, Santiago, 16 de agosto de 2004. [En línea].

ROMERO, Hugo, y Ximena TOLEDO, 2004. "Ecología urbana y gestión ambiental sustentable de las ciudades intermedias chilenas", FONDECYT 1000828. [En línea].

Entrevista al Arqto. Alberto GUROVICH W., marzo 2006. 